MRS Advances (C) 2017 Materials Research Society

DOI: $10.1557 /$ adv.2017.73

\title{
Phase Field Crystal Simulation of Grain Growth in BCC Metals during Additive Manufacturing
}

Hang Ke and Ioannis Mastorakos

Department of Mechanical and Aeronautical Engineering, Clarkson University, Potsdam, NY 13699, USA

\begin{abstract}
In this work, we demonstrate that the phase field crystal (PFC) method can be applied to identify and predict the microstructure evolution during the solidification of the BCC metals in additive manufacturing. The results reveal the columnar structure of the grains, which matches the grain growth observed in real samples produced with the additive manufacturing technique. The effect of ambient temperature, seed-seed distance and seed-seed misorientation on the grain growth has been investigated. In addition, the formation of crystal defects during the process is recorded and the resulted long-range stresses are calculated using the eigenstresses theory.
\end{abstract}

\section{INTRODUCTION}

Additive manufacturing, also known as 3D printing, is rising popularity during the last few years due to its wide applications in various industrial areas. In additive manufacturing metallic powders are deposited, one layer at the time, on a substrate bed and melted to produce the desired geometry [1]. Researchers and engineers are optimistic that this future technology can fulfill the dreams of designing and fabricating in house any part or component with the support of computer aided design (CAD) [2]. However, despite all the progress that has been made, not enough emphasis has been given to the microstructures of the build parts, which is actually very significant to the properties and performance of the final product.

The microstructure and mechanical properties of the build part can be affected by the process parameters, such as laser beam power, beam scan speed, powder feed rate, powder layer thickness and preheat temperature, during the solidification part in additive manufacturing [3]. To control and model the whole solidification process, knowing the influence of these parameters to the microstructure of the final part is really important. Many computational approaches have arisen to simulate microstructure evolution of materials. One of the most popular methods is the phase field (PF) approach. Sahoo and Chou [4] developed a PF model of the microstructure evolution of the Ti-6Al-4V alloy in electron beam additive manufacturing process. They incorporated the temperature gradient and solidification velocity as the simulation parameters, and showed that columnar dendritic spacing and width of dendrites decreases with the increase in temperature gradient and beam scan speed. Kundin et al. [5] studied the microstructure formation of Inconel 718 superalloy using PF model. They evaluated the model via comparison to Green-function calculations and Kurz-Fisher model predictions. Krivyilov et al. [6] developed a PF model of metal powder consolidation and tested with the carbonyl iron powder. Their simulations showed a good agreement with the experimental data in the consolidation time.

Recently, a new approach to PF method appeared, which is called the phase field crystal method (PFC). PFC is different from the standard PF approach since the field is only averaged in time and not in space [7], and it includes the elastic energy and symmetry properties of the 
periodic field, which results to the visualization of the periodic structure of the lattice. Moreover, PFC can characterize the systems on atomic length and diffusive time scales, which makes it faster and more efficient, compared to molecular dynamics (MD).

While PFC method has shown great advantages and has already been applied to many areas such as elastic deformation [8], grain boundaries [9] and crack propagation [10], its potential in microstructure evolution during crystal growth has not been largely explored. The goal of this paper is to simulate the microstructure evolution of the solidification process during the additive manufacturing using PFC.

The remainder of this paper is organized as follows: First, we briefly introduce the phase field crystal model for two-dimensional (2D) BCC metals and set thermodynamic parameters for it. Then, the results and discussions of the PFC simulations are shown. We studied the ambient temperature, the seed-seed distance and seed-seed orientation effects on crystal growth. In addition, the long-range stress field of the pile-up dislocations is calculated and compared. Finally, we present the conclusions of this work.

\section{THEORY}

Compared to the PF model, PFC model introduces periodic fields by including a rotationally invariant free energy scalar which is minimized by periodic solutions [11].The basic equation for PFC model was actually derived from Swift-Hohenberg ( $\mathrm{SH}$ ) equation to calculate problems in thermal convection [12]. The free energy of the model can be written as a function of the density field $\phi[13]$,

$$
F^{\prime}=\int_{V}\left\{\frac{1}{2} \phi\left[\alpha+\lambda\left(q_{0}^{2}+\nabla^{2}\right)^{2}\right] \phi+\frac{g}{4} \phi^{4}\right\} d \boldsymbol{r}
$$

In the equation, $\alpha, \lambda, q_{0}$, and $g$ are constants. The density field $\phi$ can be minimized by periodic functions for the solid state and a constant density value for the liquid state. To simplify the calculation process, equation (1) is rewritten into a dimensionless form as follows:

$$
F=\int_{V}\left\{\frac{1}{2} \psi\left[-\varepsilon+\left(1+\nabla^{2}\right)^{2}\right] \psi+\frac{1}{4} \psi^{4}\right\} d \boldsymbol{x}
$$

Where $\varepsilon=-\frac{\alpha}{\lambda q_{0}^{4}}, \psi=\phi \sqrt{\frac{g}{\lambda q_{0}^{4}}}, \mathbf{x}=q_{0} \boldsymbol{r}$, and $F=\frac{g}{\lambda^{2} q_{0}^{5}} F^{\prime}$. The non-dimensional parameter $\varepsilon$ corresponds to the ambient temperature of the simulation. The dynamics evolution of the dimensionless density field is the same as the Cahn-Hilliard model [14]:

$$
\frac{\partial \psi}{\partial t}=\Gamma \nabla^{2} \frac{\partial F}{\partial \psi}
$$

Where $\Gamma$ is the mobility constant [7] and the Gaussian random noise term is ignored. The periodic density field can be obtained from the reciprocal lattice vectors $\mathbf{G}$ by the following equation:

$$
\psi=\bar{\psi}+\sum_{G} \eta_{G} e^{i G \cdot x}+c . c .
$$


where $\bar{\psi}$ is the average density in solid state, $\eta_{G}$ is the amplitude of the reciprocal lattice vector, and c.c. represents complex conjugates. In the present work, we focus on two-dimensional (2D) systems where $\mathbf{G}$ is written as:

$$
\mathbf{G}=k_{1} \boldsymbol{q}_{1}+k_{2} \boldsymbol{q}_{2}
$$

Both $k_{1}$ and $k_{2}$ are integers. For 2D hexagonal system, the dimensionless density field is written as:

$$
\psi=\bar{\psi}+A\left[\cos (q x) \cos \left(\frac{q y}{\sqrt{3}}\right)-\frac{1}{2} \cos \left(\frac{2 q y}{\sqrt{3}}\right)\right]
$$

where $\mathrm{A}$ is a constant and $\mathrm{q}=\frac{2 \pi}{a}$. Substituting (6) into (2) and minimizing the free energy with respect to $\mathrm{A}$ and $\mathrm{q}$ leads to

$$
\bar{\psi}=\frac{\sqrt{-\varepsilon}}{2}, \mathrm{q}=\frac{\sqrt{3}}{2}, \text { and } \mathrm{A}=\frac{4}{5}\left(\bar{\psi}+\frac{1}{3} \sqrt{-15 \varepsilon-36 \overline{\psi^{2}}}\right)
$$

\section{DISCUSSION}

For the simulations, the PFC model proposed by Elder [7] has been employed. Initially, a square simulation box of size $\mathrm{N}=\mathrm{N}_{\mathrm{x}}=\mathrm{N}_{\mathrm{y}}=4096$ was used. The time step (dt) and the grid size $(\mathrm{dx}=\mathrm{dy})$ were 0.5 and $\pi / 4$ (in dimensionless units), respectively. Periodic boundary conditions have been used along all four directions. In order to prevent the grains from crossing the bottom boundary and growing at the top of the simulation box, the density field in a region of size about $3 \%$ of $\mathrm{N}$ at the top was continuously updated to the constant value of liquid state. In all simulations, the average density was $\bar{\psi}=0.285$. [15] We focus on BCC iron as an example. The microstructure evolution of the seeds was computed numerically via equations above. At the beginning of the simulations, two small circular seeds have been placed at the bottom of the simulation box. Then the crystallite grew and impinged on the other crystallite, and grain boundaries between seeds were formed. The results of the evolution of the seed growth are shown in Figure1. 

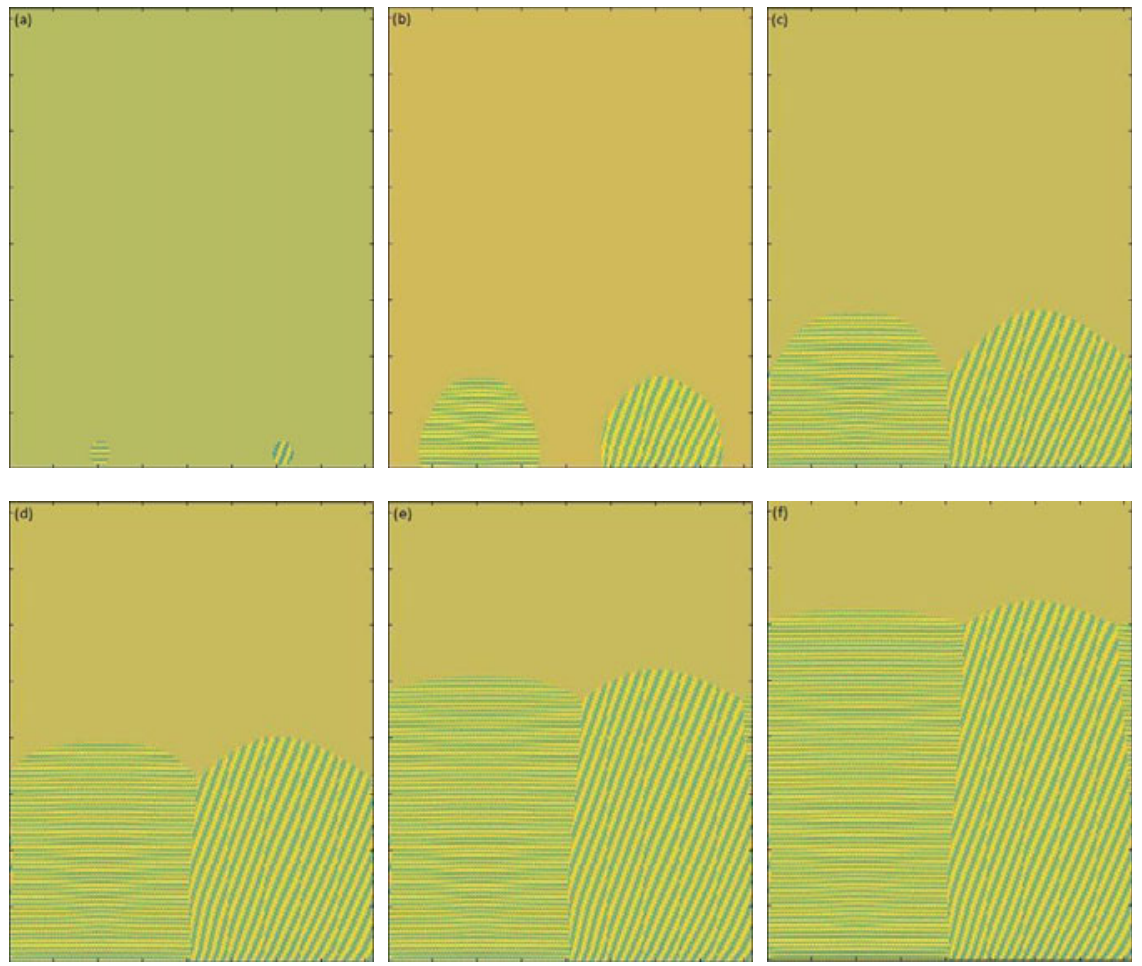

Figure 1. Time evolution of the seeds with $\varepsilon=-0.24$ at six different time steps: (a) $t=0$; (b) $t=2000 ;$ (c) $t=4000 ;$ (d) $t=6000$; (e) $t=8000$ and (f) $t=10000$.

From these graphs, we can see clearly the columnar structure, which matches the real growth in the additive manufacturing experiment [16]. Here in our simulation experiment, the length scale of the grain is about $0.1 \mu \mathrm{m}$, which is much smaller than the length scale in real samples. However, we do expect to get similar columnar grain structures if we increase the box size and allow the seeds to grow for enough time. It should also be noted that although these structures are not for pure iron but rather for Ti64 and IN718 alloys, in this work we are only focusing on the geometry of the grains rather than their structure. Furthermore, although the columnar feature may depend on the part geometries, the shape of the produced part in Ref. [16] is very similar to the shape of our simulation box, although with different dimensions. Therefore, we do not expect very different microstructure morphology (columnar structure) from Ti64 and IN718.

\section{The effect of ambient temperature}

One of the most important process parameters during the solidification is the undercooling ambient temperature, which can be denoted by the model parameter $\varepsilon$. Here we assume that the temperatures of the seeds and liquid are kept the same during the whole simulation process. The 
negative value of $\varepsilon$ can be viewed as ambient temperature which is below the melting temperature. From that point of view, a larger absolute magnitude of $\varepsilon$ signifies a lower temperature. A range of $\varepsilon$ cases it was considered, with $\varepsilon$ varying from -0.22 to -0.32 to show its effects on grain growth. The results are presented in Figure 2.
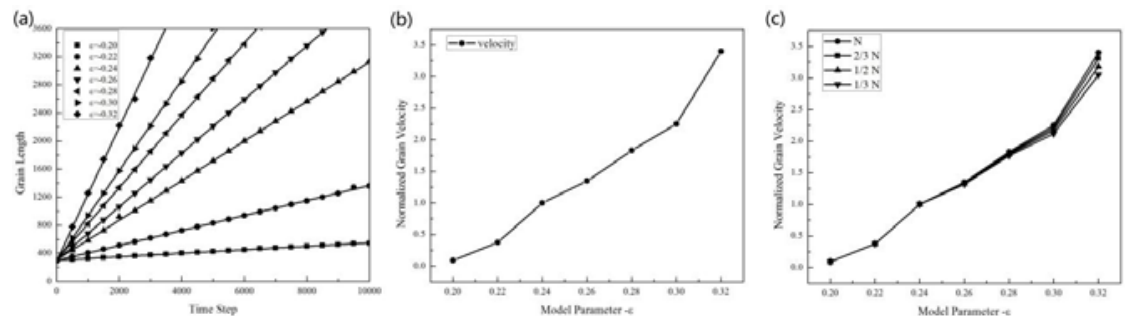

Figure 2. (a) The grain length of PFC model is compared with experiments on different model parameter $\varepsilon$, which correspond to different ambient temperature. (b) The average growth velocity is plotted as a function of model parameter $\varepsilon$, and the grain velocities are normalised by the velocity at $\varepsilon=-0.24$. (c) The seed-seed distance effects on grain growth velocity are shown by four lines, which have four different box sizes. Note that the velocities in (b) and (c) are normalised with the velocity at $\varepsilon=-0.24$.

In Figure 2a, the correlation between the average grain length as a function of the time (unitless) is shown for various temperature differences. In Figure $2 b$, the relationship between temperature difference and growth velocity (the slope of the lines in Figure2a) is plotted. It can be observed that the velocity of the grain growth is increasing in a rather linear fashion as the undercooling temperature difference $(-\varepsilon)$ is increasing. That practically means that the grain growth rate is increasing as the ambient temperature decreases, since the bigger $\varepsilon$ (absolute value) indicates a larger temperature deviation from the melting temperature.

\section{The effect of seed-seed distance}

Next, we studied the effect of the seed-seed distance on the average grain growth velocity. For that purpose, the length of the periodic box has been changed keeping the height constant and equal to $\mathrm{N}$. That way, we simulated an increase in the density of the seeds, since now there were more seeds than the original box in the same length. Three additional box sizes were considered, with length $\mathrm{N}_{x}=1 / 3 \mathrm{~N}, 1 / 2 \mathrm{~N}$ and $2 / 3 \mathrm{~N}$. Again the grain growth velocity was calculated as before. In Figure 2c, the grain growth velocity as a function of the undercooling temperature difference is plotted for all four simulation boxes. It is clearly shown that at lower negative $\varepsilon(0.20-0.26)$, e.g. at temperatures closer to the melting temperature, the seed-seed distance does not affect the velocity considerably. However, at higher negative $\varepsilon(0.28-0.32)$, e.g. lower temperatures, increasing the seed number and consequently the density of the seeds, the growth velocity decreases. This result is reasonable as with more seeds (e.g. higher seed density), the space for each one is more limited hence the growth of grains is delayed.

\section{The effect of seed orientation}

Moreover, the relative seed-seed orientation effect during the solidification process has also been studied. In two-dimensional space, the BCC lattice unit can be viewed as an equilateral 
triangle. Hence, when we apply a total lattice rotation by a $60^{\circ}$ angle, the seed orientation will remain the same. We split this difference into six angles in order to investigate the relative seed orientation effects. The results are shown in Figure 3 where the growth velocity of the grains is plotted for different seed orientations.

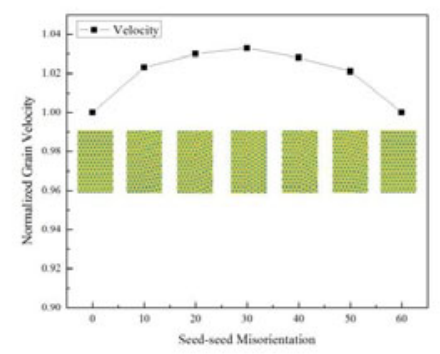

Figure 3. The relationship between growth velocity and seed orientation difference at $\varepsilon=-0.24$, and the seed-seed misorientation pictures are presented under each different angles. Note that the velocities are normalised with the velocity without see-seed misorientations. The seed orientation on the left side has not changed at all, while the seed orientation on the right side has rotated specific degrees shown below.

The results reveal a small discrepancy (of $\sim 3 \%$ ) in the growth velocities within the $60^{\circ}$ range. This difference is related to the close-packed direction with respect to the growth direction. When the close-packed direction is parallel to the growth direction, the velocity is at its minimum. When the close-packed direction is perpendicular to the growth direction, the velocity is at its maximum.

\section{Internal stresses}

Finally, the methodology introduced by Mura [17] to calculate the stress fields was used to find the stress of the grain boundaries. Due to the difference between seed orientations, grain boundaries will form during the growth of the seeds into grains. In two-dimensional analysis, the grain boundaries can be considered as a vertical pile-up of edge dislocations with a spacing that depends on the misorientation between the two adjacent grains. Therefore, a grain boundary can be modelled as a wall of edge dislocations with a constant spacing and the stress from the grain boundary can be found using the eigenstress approach. The stress is given by:

$$
\sigma_{i j}(\vec{x})=C_{i j k l} \int_{-\infty}^{\infty} \varepsilon_{l n h} C_{p q m n} G_{k p, q}\left(\vec{x}-\vec{x}^{\prime}\right) \alpha_{h m}\left(\vec{x}^{\prime}\right) d x^{\prime}
$$

Where $\sigma_{i j}, C_{i j k l}, \varepsilon_{l n h}, G_{k p, q}$ and $\alpha_{h m}$ denote the stress tensor, material stiffness tensor, permutation tensor, Green's function and dislocation density tensor respectively, and the subscript, q indicates spatial derivative with respect to spatial coordinate $x_{q}$.

The dislocations between the seeds in our cases (Figure 4) show similar patterns in Akarapu and Zbib [18]. 


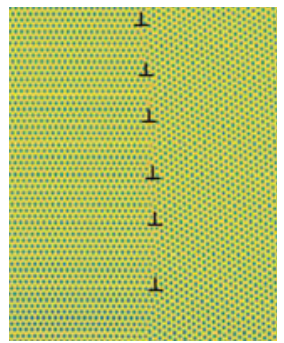

Figure 4. Dislocations at the grain boundary of two seeds with different orientations.

They derived an equation to solve (8) for isotropic materials and finite length wall, where

$$
C_{p q m n} G_{k p, q}(\bar{x})=\frac{-1}{8 \pi(1-v)}\left\{(1-2 v) \frac{\delta_{m k} \bar{x}_{n}+\delta_{n i} \bar{x}_{m}-\delta_{m n} \bar{x}_{i}}{\bar{x}^{3}}+3 \frac{\bar{x}_{n} \bar{x}_{m} \bar{x}_{i}}{\bar{x}^{5}}\right\}
$$

Where $\bar{x}$ indicates the distance away from the dislocation wall, and $v$ and $\delta$ represent Poisson ratio and Kroneker delta function respectively. Then, the stress field of the dislocation wall can be calculated by multiplying with the dislocation density tensor and integrating numerically over the length of the boundary. The long-range stress field is the sum of the stress field of each dislocation. The stresses of four different box sides $(\mathrm{N}, 2 / 3 \mathrm{~N}, 1 / 2 \mathrm{~N}$ and $1 / 3 \mathrm{~N})$ were found and compared, as well as the different seed-seed orientations. The results are shown in Figure 5.
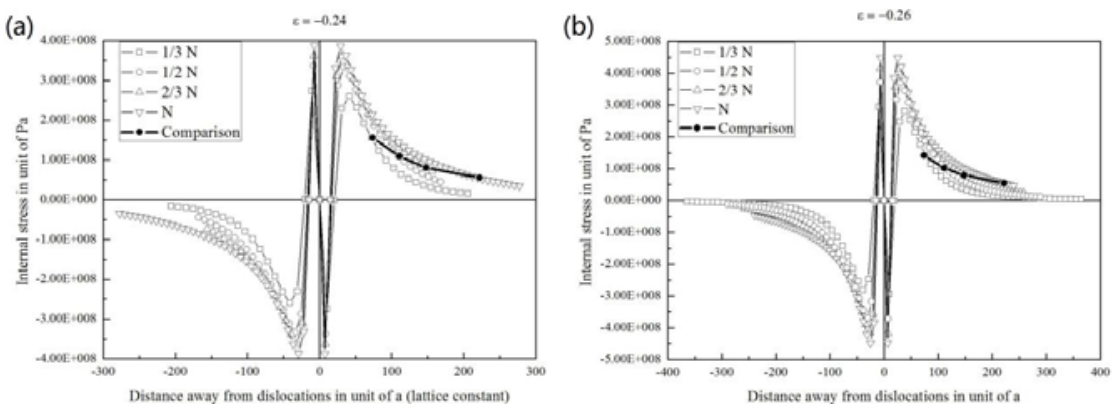


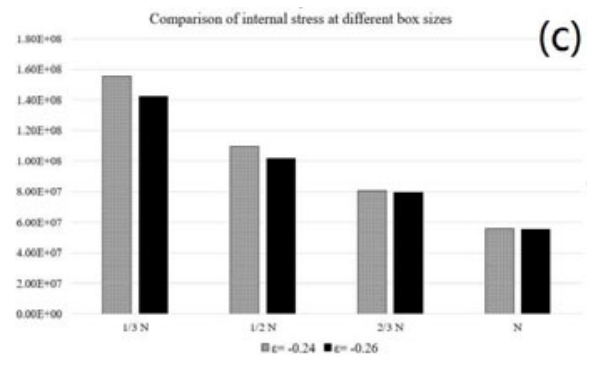

Figure 5. Comparison of internal stress of dislocations in four different box size at $\varepsilon=-0.24$ and $\varepsilon=-0.26$ are shown in (a) and (b) respectively. In addition, comparison of the internal stress at the other grain boundary is shown in (c).

In Figure $5 \mathrm{a}$ and $5 \mathrm{~b}$, the internal stress is plotted as a function of the distance from the grain boundary for four different simulation box sizes (e.g. grain densities) and two different temperatures. The internal stress fields at the other grain boundary are compared in Figure 5c. From the figure, it can be seen that the denser configurations exhibit higher internal stresses. These stresses decrease as the density of the grains is decreasing, e.g. when the grain boundaries are further apart. This can be attributed to that in larger grains there is more space for the stresses to relax. This observation suggests that in order to avoid the presence of high internal stresses inside the grains, the distance of the seeds must be kept large, or the density of the seeds relatively low. The ambient temperature also seems to have an effect at the higher densities while it becomes negligible at the small densities.

(a)

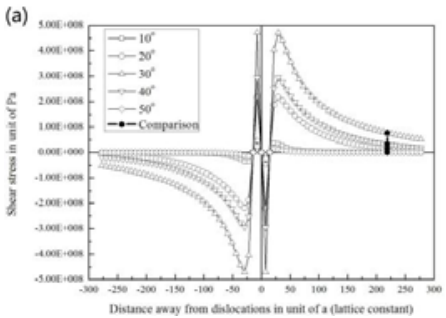

Comparison of residual stress at different seed-seed orientations

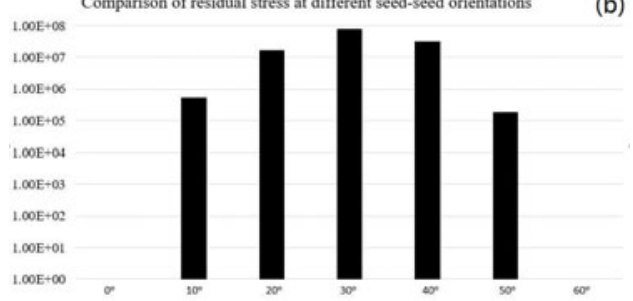

Figure 6. (a) Comparison of internal stress of dislocations at different seed-seed orientations. (b) Comparison of the internal stress at grain boundary at different seed-seed orientation. Note that the initial and final values correspond to zero misorientation that results to only one grain.

Also, the results for the seed-seed orientations in Figure 6 show that the internal stress increases with the discrepancy in their seed-seed orientations with the 30 degrees misorientation having the maximum internal stress. This change in stress is related to the incease of the dislocation density at the grain boundary due to the increase of misorientation as explained in [18]. The stresses in Figure $6 \mathrm{~b}$ follow the same trend as the velocities in Figure 3 for the same misorientation. However, the differences between the minimum and maximum values are not 
comparable. While the velocities in Figure 3 exhibit a 3\% discrepancy, in Figure $6 \mathrm{~b}$ it is of two orders of magnitude, revealing the importance of the columnar grain misorientation in the development of internal stresses inside the structure. Although the size of the grains in this work is smaller than detected in experiments, which results to larger magnitudes in stresses, the discrepancy between different orientations should remain the same since all stress - distance curves in Figure 6a follow relatively similar trends towards the large distances.

\section{CONCLUSIONS}

In conclusion, we have presented a PFC model for the atoms in the solidification process during additive manufacturing, which is one of the most promising technologies in the near future. Using PFC simulations and the solid-liquid coexistence approach, the evolution of the growth microstructure was modelled. However, since additive manufacturing is a complicated layer-by-layer process involving multiple thermal cycles and couplings of flows and physical fields, our model, in order to reduce the complexity, has not taken into account effects such as thermal convection and cooling conditions. Nevertheless, the results showing the columnar dendrite grain structure are in reasonable agreement with the experimental findings. Also, we investigated the effect of the temperature, seed misorientation and density in the grain grow velocity and the effect of the temperature to developed internal stresses. These results can allow us to eventually control and optimize the process parameters in order to improve the grain morphology for a desired shape. These findings authenticate that PFC approach can be an effective and powerful tool for quantitative simulation of microstructure evolution of solidification process during additive manufacturing.

\section{REFERENCES}

1. W.E. Frazier, J. Mater. Eng. Perform. 23, 1917 (2014).

2. K.V. Wong, A. Hernandez, K.V. Wong, and A. Hernandez, Int. Sch. Res. Not. 2012, e208760 (2012).

3. Z. Wang, T.A. Palmer, and A.M. Beese, Acta Mater. 110, 226 (2016).

4. S. Sahoo and K. Chou, Addit. Manuf. 9, 14 (2016).

5. J. Kundin, L. Mushongera, and H. Emmerich, Acta Mater. 95, 343 (2015).

6. M.D. Krivilyov, S.D. Mesarovic, and D.P. Sekulic, J. Mater. Sci. 1 (2016).

7. K.R. Elder and M. Grant, Phys. Rev. E 70, 51605 (2004).

8. N. Pisutha-Arnond, V.W.L. Chan, K.R. Elder, and K. Thornton, Phys. Rev. B 87, 14103 (2013).

9. Y.-L. Lu, T.-T. Hu, G.-M. Lu, and Z. Chen, Phys. B Condens. Matter 451, 128 (2014).

10. S. Hu, Z. Chen, Y.-Y. Peng, Y.-J. Liu, and L.-Y. Guo, Comput. Mater. Sci. 121, 143 (2016).

11. N. Provatas and K. Elder, Phase-Field Methods in Materials Science and Engineering (Wiley, Weinheim, 2010), p. 141.

12. J. Swift and P.C. Hohenberg, Phys. Rev. A 15, 319 (1977).

13. K.R. Elder, M. Katakowski, M. Haataja, and M. Grant, Phys. Rev. Lett. 88, 245701 (2002).

14. J.W. Cahn and J.E. Hilliard, J. Chem. Phys. 28, 258 (1958).

15. E. Asadi and M.A. Zaeem, JOM 67, 186 (2014).

16. Q. Jia and D. Gu, J. Alloys Compd. 585, 713 (2014). 
17. T. Mura, Micromechanics of Defects in Solids, 2nd ed. (Martinus Nijhoff Publishers, Dordrecht, 1987), p. 47.

18. S. Akarapu and H. Zbib, Int. J. Mech. Mater. Des. 4, 399 (2008). 